\title{
Mental state decoding $v$. mental state reasoning as a mediator between cognitive and social function in psychosis
}

Nicola McGlade, Caragh Behan, Judy Hayden, Therese O'Donoghue, Rosie Peel, Farhan Haq, Michael Gill, Aiden Corvin, Eadbhard O'Callaghan and Gary Donohoe

\section{Summary}

Theory of mind deficits in schizophrenia have been parsed into mental state reasoning and mental state decoding components. We report that mental state decoding as measured by the 'Eyes task' better predicted social function than mental state reasoning as measured by the 'Hinting task' in 73 out-patients with chronic schizophrenia. Mental state decoding task performance also partly mediated the influence of basic neuropsychological performance on social function. We discuss these findings in terms of the accumulating evidence that mental state decoding has particular relevance for understanding deficits in social function in schizophrenia.

\section{Declaration of interest}

None. Funding detailed in Acknowledgements.
Theory of mind is an aspect of social cognition that describes the ability to infer other people's mental state. A recent meta-analysis of almost 30 studies reported a large effect size for theory of mind impairment in schizophrenia. ${ }^{1}$ Investigations of these deficits have employed tasks that index false beliefs/deception (reasoning about the mental state of others), indirect speech (understanding irony, hints and 'faux pas'), and mental state decoding (using information such as facial expression to infer mental state). Differences between tasks (at both behavioural and neuroanatomical levels) have resulted in a distinction between mental state reasoning ability and mental state decoding ability. ${ }^{2,3}$

Following evidence linking affective recognition and social function, ${ }^{4}$ Bora $e t ~ a l^{3}$ suggested that mental state decoding may be more important than mental state reasoning for social outcome in schizophrenia. They argue that this was due to mental state decoding tasks being based on more spontaneous/automatic inferential processing than theory of mind tasks involving effortful verbal processing. Furthermore, they suggest that decoding affective states from facial expressions (e.g. eyes) is likely to be closely related to empathy and the neural network underpinning empathy (inferior frontal/anterior temporal lobe function); ${ }^{5}$ empathy has previously been shown to be important to social function in schizophrenia. ${ }^{6}$ Here we aimed to test whether mental state reasoning and mental state decoding ability differ in their ability to: (a) predict social function; and (b) mediate the relationship between neuropsychological and social function in chronic schizophrenia.

\section{Method}

After we received written informed consent, we assessed 73 patients (49 male) from a suburban Dublin psychiatric out-patient clinic using the Structured Clinical Interview for DSM-IV disorders ${ }^{7}$ to confirm diagnosis of schizophrenia or schizoaffective disorder. Criteria for participation included being aged 16-65 years, having no history of intellectual disability, acquired brain injury resulting in loss of consciousness or substance misuse in the preceding 6 months (all based on chart review). Mean age was 41.4 years, mean duration of illness was 18.2 years, and almost all were prescribed atypical antipsychotic medication. Patients' theory of mind and neuropsychological performance was compared with the normal population using a sample of 78 age- and gender-matched healthy comparison participants recruited through the local media who satisfied the inclusion criteria and were free of psychiatric illness based on clinical interview (online Table DS1).

Mental state decoding ability was measured using the revised version of the 'Reading the Mind in the Eyes' test ('Eyes task'). ${ }^{8}$ Participants were required to study 36 photographs of facial expressions and correctly identify the thought/feeling being portrayed based on four descriptors printed around each expression. Mental state reasoning ability was assessed using the 'Hinting task.' This consisted of ten vignettes, each describing a social interaction between two characters, after which the participant is required to make inferences about the intent behind a hint dropped by one of the characters.

Neuropsychological function was assessed using tests from the Wechsler Adult Intelligence Scale ${ }^{10}$ and the Cambridge Neuropsychological Test Automated Battery (CANTAB; Cambridge Cognition Ltd, Cambridge, UK), selected to index the general and specific domains of impairment commonly reported in schizophrenia, namely general cognitive function, attentional control, episodic memory and working memory (online Table DS1). ${ }^{11}$ Symptom severity was assessed using the Scale for the Assessment of Positive Symptoms ${ }^{12}$ and the Scale for Assessment of Negative Symptoms. ${ }^{13}$ Social functioning was measured using the problemsolving factor from the Independent Living Scale (ILS). ${ }^{14}$ Of the two factors yielded by the ILS (problem-solving and performance/ information), the problem-solving factor (which includes items such as 'what would you do if the taxi you ordered didn't come and it was getting late'), is reported to have good utility as a proxy measure for real-world functioning in schizophrenia. ${ }^{15}$

\section{Results}

Eyes task and Hinting task performance were significantly correlated $(r=0.389 ; P=0.001)$. Despite this, patients only performed significantly below controls on the Eyes task $(t=-2.3$; d.f. $=149$; $P=0.023$ ). Eyes task performance correlated with verbal and performance IQ, and verbal and spatial working memory $(P=0.003-0.000006)$ but not with verbal episodic memory. Hinting task performance by contrast was correlated with verbal episodic and working memory $(P=0.008-0.02)$ but not IQ or spatial memory performance. Neither task correlated with attention control. Eyes task performance alone was moderately negatively correlated with positive symptom severity $(r=-0.25$; 
$P=0.03)$ but not negative symptom severity. Neither task was associated with age, gender, duration of illness or medication dosage.

Scores on the ILS were significantly positively associated with Eyes task performance $(r=0.41 ; P=0.001)$ but not with Hinting task performance $(P>0.05)$. To test whether social functioning was predicted by mental state decoding performance (Eyes task performance) we performed a multiple regression analysis with ILS scores as the dependent variable. Symptom severity, which was significantly correlated with social function, was entered in the first step of the analysis, explaining $26.5 \%$ of variance in ILS scores $(20 \%$ of which was contributed by negative symptom severity alone). This was followed by scores on the Eyes task and Hinting tasks on the second step. Eyes task performance explained a further $9.0 \%$ of the variance in social functioning $\left(F_{1,63}\right.$ change $=8.5$; $P=0.005$ ); Hinting task performance was not a significant variable in the regression equation. The statistical significance of these results was unchanged by considering patients with schizophrenia and patients with schizoaffective disorder separately.

We performed two further stepwise multiple regression analyses. In the first analysis, when verbal IQ (the neuropsychological measure most highly correlated with ILS performance) was entered on the first step of the analysis followed by Eyes task performance on the second step, Eyes task performance continued to significantly predict variance in ILS $\left(r^{2}\right.$ change $=0.09, F_{1,68}=7.26$, $P=0.009$ ). In the second regression analysis, when Eyes task performance was entered on the first step and verbal IQ on the second, verbal IQ's ability to predict ILS scores was partly attenuated $\left(r^{2}\right.$ change reducing from $16.3 \%$ to $\left.7.5 \%\right)$. Following Baron \& Kenny's criteria ${ }^{16}$ for mediating variables this suggests that Eyes task performance partly mediated the relationship between verbal IQ and ILS scores. Again, the statistical significance of these results was unchanged by considering patients with schizophrenia and patients with schizoaffective disorder separately.

\section{Discussion}

This study provides further evidence that mental state decoding rather than mental state reasoning ability predicts social function in patients with chronic schizophrenia. Bora et $\mathrm{l}^{3}$ argue that the mental state decoding requires emotional perception and empathy. Perceptual deficits in social cognition (e.g. affect recognition) are reported as stable over time in schizophrenia, apparent in both patients with first-episode schizophrenia and those who are chronically ill, and mediate the relationship between basic cognition and social function. ${ }^{4}$ Our findings suggest that decoding the mental state of others is similarly important to social function in schizophrenia. This view is consistent with the model of emotional intelligence developed by Mayer \& Salovey ${ }^{17}$ in which emotional perception and emotional understanding are two of the four main abilities related to 'emotional intelligence' (together with emotional self-regulation and emotional facilitation of thinking), which contributes to higher social functioning.

Because of the general decline in cognitive function associated with schizophrenia, a challenge for studies of specific cognitive functions (either basic or social) is to establish whether any specific deficit has a unique or particular value in explaining outcome. In the regression analyses undertaken, mental state decoding performance (on the Eyes task) was able to explain variance in social function even after the variance explained by general cognitive ability (measured by verbal IQ) was already accounted for. Furthermore, accounting for the variance in mental state decoding led to attenuation in the amount of variance explained by general cognition. These results lead us to conclude that mental state decoding is not simply reflecting more general aspects of cognitive decline in schizophrenia, but instead is important in its own right in explaining social function. As further confirmation of these findings, and the earlier report by Bora et $a l,{ }^{3}$ it may be helpful to investigate this relationship between mental state decoding and social function using multiple mental state decoding tasks within the same study design. Finally, for future studies, the ILS measure employed here, although previously validated in schizophrenia, ${ }^{15}$ is a proxy measure for social function and outcome; further theory of mind studies may benefit from more direct outcome measures.

\section{Nicola McGlade, MLitt, Caragh Behan, MB, MRCPsych, Cluain Mhuire Family Centre, Blackrock, Co. Dublin; Judy Hayden, BA, Therese O'Donoghue, MSC, Rosie Peel, HDipPsych, Neuropsychiatric Genetics Research Group, Trinity College Dublin; Farhan Haq, MB, MRCPsych, Cluain Mhuire Family Centre, Blackrock, Co. Dublin; Michael Gill, Haq, MB, MRCPSych, Cluain Mhuire Family Centre, Blackrock, Co. Dublin; Michael Gill,
MRCPsych, MD, Aiden Corvin, MRCPsych, PhD, Neuropsychiatric Genetics Research Group, Trinity College Dublin; Eadbhard O'Callaghan, MRCPsych, MD, Cluain Mhuire Family Centre, Blackrock, Co. Dublin; Gary Donohoe, PhD, Neuropsychiatric} Genetics Research Group, Trinity College Dublin, Ireland

Correspondence: Dr Gary Donohoe, Neuropsychiatric Genetics Research Group, Department of Psychiatry, Trinity Health Sciences Building, St. James's Hospital, Dublin 8, Ireland. Email: donoghug@tcd.ie

First received 20 Aug 2007, final revision 3 Mar 2008, accepted 20 Mar 2008

\section{Acknowledgements}

This research was supported by research grants from the Wellcome Trust (M.G., A.C.), science Foundation Ireland (G.D.) and the Higher Education Authority of Ireland (M.G.)

\section{References}

1 Sprong $\mathrm{M}$, Schothorst $\mathrm{P}$, Vos $\mathrm{E}$, Hox J, van Engeland $\mathrm{H}$. Theory of mind in schizophrenia: meta-analysis. Br J Psychiatry 2007; 191: 5-13.

2 Shamay-Tsoory SG, Shur S, Barcai-Goodman L, Medlovich S, Harari H, Levkovitz Y. Dissociation of cognitive from affective components of theory of mind in schizophrenia. Psychiatry Res 2007; 149: 11-23.

3 Bora E, Eryavuz A, Kayahan B, Sungu G, Veznedaroglu B. Social functioning, theory of mind and neurocognition in outpatients with schizophrenia; mental state decoding may be a better predictor of social functioning than mental state reasoning. Psychiatry Res 2006; 145: 95-103.

4 Addington J, Saeedi H, Addington D. Influence of social perception and social knowledge on cognitive and social functioning in early psychosis. Br J Psychiatry 2006; 189: 373-8.

5 Sabbagh MA, Moulson MC, Harkness KL. Neural correlates of mental state decoding in human adults: an event-related potential study. J Cogn Neurosci 2004; 16: 415-26.

6 Shamay-Tsoory SG, Shur S, Harari H, Levkovitz Y. Neurocognitive basis of impaired empathy in schizophrenia. Neuropsychology 2007; 4: 431-8.

7 First MB, Spitzer RL, Gibbon M, Williams BW. Structural Clinical Interview for DSM-IV Axis I Disorders. American Psychiatric Press, 1996.

8 Baron-Cohen S, Wheelwright S, Hill J, Raste Y, Plumb I. The "Reading the Mind in the Eyes" Test revised version: a study with normal adults, and adults with Asperger syndrome or high-functioning autism. J Child Psychol Psychiatry 2001; 42: 241-51.

9 Corcoran R, Mercer G, Frith CD. Schizophrenia, symptomatology and social inference: investigating 'theory of mind' in people with schizophrenia. Schizophr Res 1995; 17: 5-13.

10 Wechsler D. Wechsler Adult Intelligence Scale (3rd edn). Psychological Corporation, 1997.

11 Donohoe G, Clarke S, Morris D, Nangle JM, Schwaiger S, Gill M, Corvin A, Robertson $\mathrm{IH}$. Are deficits in executive sub-processes simply reflecting more general cognitive decline in schizophrenia? Schizophr Res 2006; 85: 168-73.

12 Andreasen NC. The Scale for the Assessment of Positive Symptoms (SAPS). University of lowa, 1983

13 Andreasen NC. Negative symptoms in schizophrenia. Definition and reliability. Arch Gen Psychiatry 1982; 39: 784-8.

14 Loeb PA. ILS: Independent Living Scales Manual. Psychological Corporation, 1996.

15 Revheim N, Medalia A. The Independent Living Scales as a measure of functional outcome for schizophrenia. Psychiatr Serv 2004; 55: 1052-4

16 Baron RM, Kenny DA. The moderator-mediator variable distinction in social psychological research: conceptual, strategic, and statistical considerations. J Pers Soc Psychol 1986; 51: 1173-82.

17 Mayer JD, Salovey P. What is emotional intelligence? In Emotional Development and Emotional Intelligence: Implications for Educators (eds P Salovey \& D Sluyter): 3-31. Basic Books, 1997. 